\title{
Strong diffusional coupling between spines and dendrites promotes long-term potentiation of Schaffer collateral synapses
}

\author{
Shuting Yin, Christian Schulze \& Thomas G. Oertner* \\ Institute for Synaptic Physiology, Center for Molecular Neurobiology (ZMNH), University Medical Center \\ Hamburg-Eppendorf (UKE), Falkenried 94, 20251 Hamburg, Germany \\ *Corresponding author: \\ Thomas G. Oertner \\ Phone: +49 40741058228 \\ Fax: +4940741058364 \\ Email: thomas.oertner@zmnh.uni-hamburg.de
}

\begin{abstract}
Dendritic spines on CA1 pyramidal cells are highly variable in size and shape. For some spine synapses, long and narrow spine necks provide strong diffusional and electrical isolation from the main dendrite, and it has been speculated that synapses on well isolated spines could be more plastic than synapses on spines with low resistance necks. Here we test this hypothesis by first measuring the diffusional resistance of spine necks, then pairing two-photon glutamate uncaging with bursts of back-propagating action potentials. Paired stimulation induced significant (155\%) long-term potentiation (LTP) of synapses on weakly isolated spines, but no net functional change of synapses on highly isolated spines. No correlation was found between spine head volume and functional plasticity of the synapses. We conclude that contrary to our expectations, diffusional isolation makes potentiation of synapses more difficult. Our results support the concept that delivery of plasticity-related proteins from the dendrite into the spine is a limiting factor for LTP.
\end{abstract}




\section{Introduction}

The anatomical diversity of dendritic spine shapes has been the subject of intense curiosity for a century. The concept of biochemical compartmentalization in mushroom-shaped spines has been validated in numerous functional imaging studies $(1,2)$. The relatively long residence time of activated enzymes in spines may keep biochemical reactions (e.g. phosphorylation events) restricted to active synapses. In recent years, evidence has accumulated that spines with long and thin necks experience strong depolarization of the head during synaptic transmission $(3,4)$. This strong depolarization leads to efficient unblocking of NMDA receptors and boosts calcium influx into the spine head (5-7). As high calcium concentrations are a key requirement for long-term potentiation (LTP), it is tempting to speculate that synapses on well isolated spines could have a lower threshold for LTP induction. On the other hand, successful potentiation requires an enlargement of the postsynaptic density (PSD) and insertion of additional glutamate receptors into the PSD $(8,9)$. Recent studies suggest that these receptors and associated scaffolding proteins are delivered from the dendrite into the spine $(10,11)$. It is therefore conceivable that a narrow spine neck, by restricting diffusional access to the spine, would make potentiation of the synapse more difficult (12). Whether and in which direction spine neck geometry affects the plasticity of excitatory synapses can only be determined experimentally, by coincident activation of single synapses on spines with known diffusional properties.

Spine neck dimensions are well below the resolution limit of light microscopy and thus difficult to measure in live tissue. Even state-of-the-art super-resolution approaches (13) suffer from poor resolution along the optical axis. If the measurement precision is not isotropic, the true length of the spine neck will be underestimated by a variable factor that depends on the orientation of the spine in the tissue. Furthermore, due to their variable internal organization (actin bundles, endoplasmic reticulum), even spine necks with identical outer dimensions might have different mean free path lengths for diffusing molecules (14). For our study, we did not attempt to determine the outer dimensions of spines, but instead measured the time constant of diffusional equilibration between spine head and dendrite in unperturbed neurons, before patch-clamp recordings. We compared two-photon photoactivation and fluorescence recovery after photobleaching (FRAP); both methods produced reproducible and internally consistent results.

To stimulate individual, identified synapses in a precisely timed manner, two-photon uncaging of glutamate is the method of choice. For successful LTP induction, strong $\mathrm{Ca}^{2+}$ influx is essential, and extracellular solutions with low or zero $\mathrm{Mg}^{2+}$ are usually employed to unblock NMDA receptors during plasticity induction $(2,15)$. This experimental trick disables the coincidence detector function of NMDA receptors. Under physiological conditions, simultaneous depolarization of the postsynaptic neuron is a necessary condition for NMDA receptor-mediated $\mathrm{Ca}^{2+}$ influx. Here we used artificial cerebrospinal fluid (ACSF) containing $1 \mathrm{mM} \mathrm{Mg}^{2+}$ to keep coincidence detection intact (16). We combined bursts of backpropagating action potentials (bAPs) in the postsynaptic neuron with two-photon glutamate uncaging to induce plasticity at spine synapses in CA1. The outcome of this pairing protocol was quite variable: Some synapses were significantly strengthened; others only weakly affected or even depressed. In every plasticity experiment, we also determined the diffusional resistance of the spine neck. To our surprise, synapses on highly isolated spines rarely changed their strength in response to the pairing protocol while 
synapses on spines with low resistance necks were much more likely to become potentiated. Thus, we can rule out the hypothesis that a high spine neck resistance promotes synaptic potentiation. Our data suggest that a long and narrow spine neck has a stabilizing effect on the weight of the resident synapse.

\section{Results}

Measuring diffusional coupling between CA1 dendrites and spines. CA1 pyramidal cells in organotypic slices from rat hippocampus were biolistically transfected with expression vectors encoding for the red fluorescent protein tdimer2 and photoactivatable GFP (PA-GFP, Fig. 1a). After 2-3 weeks of expression, we characterized the diffusional coupling between a number of individual spines and their parent dendrite using photoactivation of PA-GFP $(810 \mathrm{~nm})$ and fluorescence recovery after photobleaching (FRAP) of tdimer2 (Fig. $1 \mathrm{~b}$ and c). The time constants were extracted by fitting a single exponential function to the time course of spine fluorescence after the laser pulse (Fig. 1d). We found that $\tau_{\text {decay }}$ of PA-GFP and $\tau_{\text {recovery }}$ of tdimer 2 were highly correlated in individual spines (Fig. 1e), indicating that either measure provided us with reliable information about diffusional coupling between spine and dendrite. We used the average of $\tau_{\text {decay }}$ and $\tau_{\text {recovery }}$ as a robust measure of diffusional coupling ( $\left.\tau_{\text {equ }}\right)$. Most spines were strongly coupled to the dendrite (small $\tau_{\text {equ }}$ ), highly isolated spines were quite rare. As we were interested in the functional consequences of strong diffusional isolation, we preferentially selected spines with large $\tau_{\text {equ }}$ for subsequent functional characterization and plasticity experiments.

Induction of LTP by pairing glutamate uncaging with postsynaptic bursts. After selecting a spine for plasticity induction, we perfused MNI-caged-L-glutamate $(2.5 \mu \mathrm{M})$ and established whole-cell patch clamp. The red cytoplasmic fluorescence ( $980 \mathrm{~nm}$ excitation) was used to visualize dendritic morphology and to position the uncaging spot $0.5 \mu \mathrm{m}$ from the edge of the selected spine. After break-in, we immediately started to uncage glutamate with short pulses from a second Ti:Sapph laser $(720 \mathrm{~nm}, 0.5$ $\mathrm{ms})$. We determined the baseline potency of the synapse by collecting a series of uncaging-evoked postsynaptic currents (uEPSCs) at a rate of $1 / \mathrm{min}$. After collecting baseline uEPSCs ( $\sim 15$ min after breakin), we paired uncaging pulses with depolarizing current injections $(100 \mathrm{~ms})$ to generate bursts of 7-9 back-propagating action potentials (bAPs). Paired stimulation was repeated 20 times. Synaptic potency was assessed again $30 \mathrm{~min}$ after the pairing protocol by a second set of uncaging pulses (Fig. 2a). Experiments were classified as significant LTP, LTD, or 'no change' based on the outcome of t-tests. In a first set of experiments, the pairing stimulation was repeated at $0.1 \mathrm{~Hz}$. In about half of the experiments, this protocol induced a significant change in potency of the 'paired' synapse. A stronger protocol with 20 pairings delivered at $0.5 \mathrm{~Hz}$ produced an almost identical outcome (chi squared test, $p=0.8$ ), suggesting that only about half of spine synapses were plastic.

As a control experiment, we assessed the stability of synaptic potency over 30 min with no stimulation (Fig. 2b). As expected, the majority of synapses (72\%) maintained their strength during the $30 \mathrm{~min}$ (Fig. 2D), indicating that spontaneous changes in synaptic potency were rare. As an additional control, we tested whether postsynaptic spike bursts alone (20 bAP bursts at $0.5 \mathrm{~Hz}$ ) would affect synaptic potency (Fig. 2c). There was no difference in the outcome distributions of the no stimulation and bAP groups (chi squared test, $p=0.2$, Fig. 3a). The distribution of the paired experiments, however, was different from the control experiments $(p=0.0001)$. 
Without postsynaptic stimulation, UEPSC amplitudes before and after the waiting period (30 min) were highly correlated $\left(r^{2}=0.84\right.$, Fig. $\left.3 b\right)$. Twenty trains of bAPs without simultaneous uncaging induced some plasticity, but the correlation of UEPSC amplitudes before and 30 min after bAP bursts was still high $\left(r^{2}=\right.$ 0.60). Paired stimulation destroyed the correlation between baseline uEPSC amplitudes and final potencies $\left(r^{2}=0.19\right)$. There was no simple relationship between initial synaptic potency and the direction or magnitude of plasticity.

Well isolated synapses are less plastic. We were surprised by the fact that many spine synapses did not change their potency when challenged with repeated paired stimulations. We speculated that the degree of diffusional isolation from the dendrite could affect the plasticity of individual synapses.

Analogous to an electrical RC circuit, the time constant of equilibration between spine and dendrite ( $\left.\tau_{\text {equ }}\right)$ depends on the diffusional resistance of the spine neck $\left(R_{n}\right)$, the size of the reservoir (spine head volume, $V)$, and the diffusion coefficient of the fluorescent molecule $(D)(17)$ :

$$
\tau=\frac{R_{n e c k} V}{D}
$$

therefore

$$
R_{n e c k}=\frac{\tau D}{V}
$$

The diffusion coefficient $D$ has been experimentally measured in dendritic cytoplasm (18), but it is unclear whether cytoplasmic viscosity is identical in dendrites and spines. What we know is that the diffusional resistance of the spine neck is proportional to the measured parameters $\tau$ and $V$ :

$$
R_{n e c k} \sim \frac{\tau}{V}
$$

We therefore decided to report $\tau / V$ as a measure of spine neck resistance, in units of $\left[\mathrm{s} / \mu \mathrm{m}^{3}\right]$. The median $R_{\text {neck }}$ in our sample was $3.58 \mathrm{~s} / \mu \mathrm{m}^{3}$, meaning it took $358 \mathrm{~ms}$ to exchange $63 \%$ (1/e) of the fluorescent molecules in a typical spine $\left(0.1 \mu \mathrm{m}^{3}\right)$. The range was large, from $0.7 \mathrm{~s} / \mu \mathrm{m}^{3}$ to $15 \mathrm{~s} / \mu \mathrm{m}^{3}$, suggesting highly variable neck geometries.

Performing plasticity experiments after measuring diffusional coupling allowed us to test for correlations between the amount of plasticity induced at individual 'paired' synapses and the diffusional resistance of the spine neck (Fig. 4a). In a total of 28 pairing experiments, 13 synapses did not significantly change their strength (open markers, $p>0.05$ ). Surprisingly, very strong potentiation ( $>200 \%$ ) was seen in spines with relatively low neck resistance (filled markers). In the two sets of control experiments, no stimulation and bAP bursts only, spontaneous changes in synaptic strength (Fig. 4b, filled markers) were not correlated with spine neck resistance. On average, there was no net potency change in the group of unstimulated spines $(106 \% \pm 6 \%, n=7)$ and in the group that was invaded by bAP bursts $(102 \% \pm 8 \%, n=$ 18, Fig. 4c). Therefore, we pooled these two groups ('no stim' and 'bAPs only') to form a single control group. 
To compare the amount of plasticity induced at synapses on low and high neck resistance spines, we split the population of paired spines at the median spine neck resistance $3.58 \mathrm{~s} / \mathrm{\mu m}^{3}$ (Fig. $4 \mathrm{~d}$ ). We found that spine synapses with low neck resistance were significantly potentiated compared to controls ( $155 \% \pm$ $19 \%, p=0.01$, ANOVA followed by t-tests), while spine synapses with high neck resistance were not $(103 \% \pm 12 \%, p=0.99)$.

As we calculated spine neck diffusional resistance from the time constant of equilibration $\left(\tau_{\text {equ }}\right)$ and the spine head volume, we asked whether one of these directly measured parameters could already explain the differences in plasticity. However, splitting the population of paired spines at the median $\tau_{\text {equ }}$ did not reveal a significant difference in plasticity between the two groups (Fig. 4e, $p=0.8$ ), and neither did spine head volume $(p=0.2)$. Thus, spine neck resistance was the only structural parameter correlated with synaptic plasticity, albeit not in the direction we expected.

We were concerned that the differences in plasticity might be due to other factors than $R_{\text {neck. }}$. Did we stimulate one set of spines more strongly? The laser pulse used for glutamate uncaging bleaches some of the red fluorescent protein in the spine. The degree of bleaching, which is a measure of local two-photon activation and therefore proportional to the amount of glutamate released, was not different between the two groups (low $R_{\text {neck }}$ group: $23 \% \pm 4 \%$ bleach, high $R_{\text {neck }}$ group: $25 \% \pm 4 \%$ bleach, $p=0.7$ ), indicating stimulation strength was not systematically different. Was wash-out different in the two groups? The time between break-in and start of the induction protocol was not different between groups (low $R_{\text {neck }}$ group: $17 \pm 2 \mathrm{~min}$, high $R_{\text {neck }}$ group: $15 \pm 1 \mathrm{~min}, p=0.4$ ), excluding wash-out as a possible explanation for the observed difference in plasticity. Is it possible that synapses on high $R_{\text {neck }}$ spines were stronger to begin with and therefore more difficult to potentiate? There was no difference in initial potency (low $R_{\text {neck }}$ group: $19.6 \pm 2.1 \mathrm{pA}$, high $R_{\text {neck }}$ group: $\left.20.4 \pm 1.3 \mathrm{pA}, p=0.7\right)$. Spine head volumes, however, were significantly larger in low $R_{\text {neck }}$ spines (low $R_{\text {neck}}: 0.18 \pm 0.03 \mu \mathrm{m}^{3}$, high $R_{\text {neck }}$ group: $0.08 \pm 0.01 \mu \mathrm{m}^{3}, p=$ 0.003 ). Consistent with this finding, a positive correlation between spine head volume and spine neck diameter was described in earlier two-photon studies (19) and in a recent serial EM study of perfusionfixed hippocampal tissue (20). Thus, even though spine neck geometry is not completely independent of spine head size, we found that only $R_{\text {neck }}$ had a measurable impact on synaptic plasticity.

\section{Discussion}

Our pairing protocol had overall a relatively modest effect on synaptic strength. To maintain coincidence detection by NMDA receptors, we used ACSF containing $1 \mathrm{mM} \mathrm{Mg}^{2+}$ and paired uncaging pulses with brief bursts of postsynaptic action potentials. Under these conditions, calcium influx is boosted and plasticity is triggered if the first back-propagating AP arrives within 40-50 ms after the $\operatorname{EPSP}(6,23)$. Until now, many studies have used glutamate uncaging in magnesium-free solution $(2,15,21,22)$. This protocol leads to strong $\mathrm{Ca}^{2+}$ influx and LTP at every stimulated spine. However, the potential influence of the spine neck on spine head depolarization cannot be investigated with uncaging in zero $\mathrm{Mg}^{2+}$ since this circumvents the requirement for coincidence detection by NMDA receptors. In physiological $\mathrm{Mg}^{2+}$, the calcium rise induced by the interaction of back-propagating APs and glutamate receptors is supralinear and most pronounced in highly isolated spines. We therefore expected that spines with high $R_{\text {neck }}$ would be more likely to undergo LTP and were surprised by the lack of positive correlation. It should be 
noted that we actively biased our sample towards highly isolated spines, which are relatively rare across the population. A 'blind' electrophysiological LTP experiment would therefore be dominated by synapses on low $R_{\text {neck }}$ spines, which did produce substantial (155\%) potentiation in our experiments. We performed somatic voltage-clamp recordings to measure uncaging-evoked synaptic currents, but acknowledge the fact that spine heads escape the voltage clamp when responding to glutamate $(5,24)$. Since we were interested in changes before and after pairing rather than determining the absolute number of AMPA receptors in a synapse, we considered it important to keep the dendritic membrane potential as constant as possible over the entire experiment ( $\sim 5 \mathrm{~min}$ ). A consequence of escaping (i.e. depolarizing) spine heads is that the measured LTP may underestimate the true increase in synaptic conductance.

Our results challenge the widely held belief that spine necks have evolved to enhance synaptic plasticity (19). The high input resistance and biochemical isolation, it was argued, would trap calcium-activated enzymes such as CaMKII close to their targets at the synapse, facilitating LTP. Our data suggest this trapping effect is not sufficient to enable LTP at the most isolated synapses. Rather the opposite seems true, and less isolated spines are more likely to increase their AMPA currents after pairing. The major delivery route for spine AMPA receptors is lateral diffusion in the membrane rather than constitutive exocytosis in the spine head itself. The spine neck restricts diffusion of receptors and other membrane proteins into and out of mushroom spines $(12,25)$. Restricted access to AMPA receptors and other plasticity-related proteins is a plausible explanation for our finding that synapses on highly isolated spines were less plastic.

\section{Materials and Methods}

Organotypic hippocampal cultures. Hippocampal slice cultures were prepared in accordance with the ethical standards of the German Animal Welfare Act and the State Authority of Hamburg, Germany, using female rat pups (Wistar) at postnatal day 5-6. The hippocampus was dissected as described (28) and cut in $400 \mu \mathrm{m}$ thick slices using a Mcllwain tissue chopper. No antibiotics were used during the preparation or in the culture medium. At DIV 7-9, cultures were transfected with a 1:1 mixture of expression vectors encoding tdimer2 (29) and PA-GFP (30), each driven by the synapsin-1 promoter, using a Helios gene gun (Bio Rad).

Two-photon imaging and photoactivation. After 2-3 weeks of expression (DIV 24-30), photoactivation experiments were performed on a custom-built two-photon microscope controlled by Scanlmage 3.8 open source software (31). We extended the capabilities of Scanlmage to perform user-defined curved line scans and automatic drift correction. Two Ti:Sapph lasers (MaiTai DeepSee, Spectra-Physics) were coupled into the microscope through a polarizing beamsplitter cube (Thorlabs), controlled by electrooptical modulators (Conoptics) for simultaneous imaging $(980 \mathrm{~nm})$, photoactivation $(810 \mathrm{~nm})$ or uncaging $(720 \mathrm{~nm}$ ) of MNI-caged-L-glutamate (Invitrogen). Photons were collected through objective (60x $0.9 \mathrm{NA}$, Olympus) and condenser (1.4 NA, Olympus) and detected with 4 GaAsP PMTs (Hamamatsu). During imaging and plasticity induction, cultures were submerged in artificial cerebrospinal fluid (ACSF) 
containing (in mM): $127 \mathrm{NaCl}, 2.5 \mathrm{KCl}, 2 \mathrm{CaCl}_{2}, 1 \mathrm{MgCl}_{2}, 25 \mathrm{NaHCO}_{3}, 1.25 \mathrm{NaH}_{2} \mathrm{PO}_{4}, 25 \mathrm{D}$-glucose, 2.5 MNI-caged-L-glutamate ( $\mathrm{pH} 7.4, \sim 308 \mathrm{mOsm}$, saturated with $95 \% \mathrm{O}_{2} / 5 \% \mathrm{CO}_{2}$ ).

Spine head volume measurements. We imaged spines in stratum radiatum, located on thin oblique dendrites of CA1 pyramidal cells. We used the integrated fluorescence of the spine head as a measure of spine volume. Spine fluorescence $\left(F_{\text {spine }}\right)$ was determined from a stack of images and normalized by the maximum fluorescence from the same cell $\left(F_{\text {max }}\right)$, obtained by immersing the point spread function (PSF) in the trunk of the apical dendrite. The volume of the PSF $\left(V_{\mathrm{PSF}}=0.18 \mathrm{fl}\right)$ was determined from images of $0.1 \mu \mathrm{m}$ fluorescent microspheres (Molecular Probes) excited at $980 \mathrm{~nm}$. The volume of the spine head $\left(V_{\text {spine }}\right)$ was calculated as

$$
V_{\text {spine }}=\frac{V_{P S F} F_{\text {spine }}}{F_{\max }}
$$

Electrophysiology and pairing protocol. Patch-clamp recordings were performed at $32^{\circ} \mathrm{C}$ under Dodt contrast using a Multiclamp 700B amplifier (Axon Instruments) and Ephus open source software (32). Patch pipettes were filled with pipette solution consisting of (in $\mathrm{mM}$ ): $135 \mathrm{~K}$-gluconate, $4 \mathrm{MgCl}_{2}, 4 \mathrm{Na}_{2^{-}}$ ATP, 0.4 Na-GTP, $10 \mathrm{Na}_{2}$-phosphocreatine, 3 ascorbate, and 10 HEPES (pH 7.2, 295 mOsm). The power of the $720 \mathrm{~nm}$ uncaging laser was adjusted to bleach $25 \%$ of red spine fluorescence during a $1 \mathrm{~ms}$ pulse directed to the outer edge of a test spine. The test spine was not used for physiological experiments. LTP was induced by pairing of $0.5 \mathrm{~ms}$ uncaging pulses with $100 \mathrm{~ms}$ depolarizing current injections (20 repeats at $0.1 \mathrm{~Hz}$ or $0.5 \mathrm{~Hz}$ ). The potency of the synapse was assessed 30 min after the pairing protocol (5 test pulses, $1 / \mathrm{min}$ ). Input and access resistance were continuously monitored; experiments with $>50 \%$ change of either value were excluded from further analysis. As a control group, we assessed the stability of synaptic responses on spines that were invaded by bAPs, but not simultaneously activated by glutamate uncaging. Some of these spines were on neurons where no spine received paired stimulation $(n=10)$, others on the same dendrite as a paired spine, but at $>10 \mu \mathrm{m}$ distance $(n=8)$.

Data analysis. Analysis of line scan data and curve fitting was performed with custom software written in Matlab. We used GraphPad Prism for statistical analysis. Long-term plasticity of single synapses was assessed by comparing uEPSP amplitudes at baseline to uEPSP amplitudes 30 min after pairing (Student's $t$-test, two-tailed). On the population level, significance was tested by Chi squared tests or by ANOVA followed by two-tailed Student's $t$-tests. We considered $p<0.05$ as significant $\left({ }^{*}\right)$ and $p<0.01$ as highly significant $\left({ }^{* *}\right)$. Data are shown as mean \pm standard error (SEM).

Author contributions. S.Y. performed experiments and analyzed data, C.S. developed software for the laser scanning microscope, T.G.O. designed the study and wrote the manuscript.

Competing interests statement. The authors declare no competing interests.

Data Availability. The authors will make available all data and materials. Interested parties should contact T.G.O. (thomas.oertner@zmnh.uni-hamburg.de) to make requests. 


\section{Acknowledgements}

We thank Dr. Christine E. Gee for critical reading of the manuscript and Iris Ohmert for excellent technical support. This study was supported by the German Research Foundation (DFG) through Priority Programme SPP 1665, Collaborative Research Center SFB 936, Research Unit FOR 2419, and by the Ministry of Science, Research and Equalities Hamburg (Z-FR LF).

\section{References}

1. Sabatini BL, Oertner TG, Svoboda K (2002) The life cycle of $\mathrm{Ca}(2+)$ ions in dendritic spines. Neuron 33(3):439-452.

2. Lee SJ, Escobedo-Lozoya Y, Szatmari EM, Yasuda R (2009) Activation of CaMKII in single dendritic spines during long-term potentiation. Nature 458(7236):299-304.

3. Harnett MT, Makara JK, Spruston N, Kath WL, Magee JC (2012) Synaptic amplification by dendritic spines enhances input cooperativity. Nature 491(7425):599-602.

4. Bloodgood BL, Giessel AJ, Sabatini BL (2009) Biphasic synaptic Ca influx arising from compartmentalized electrical signals in dendritic spines. PLoS Biol 7(9):e1000190.

5. Grunditz A, Holbro N, Tian L, Zuo Y, Oertner TG (2008) Spine Neck Plasticity Controls Postsynaptic Calcium Signals through Electrical Compartmentalization. J Neurosci 28(50):13457-13466.

6. Holbro N, Grunditz A, Wiegert JS, Oertner TG (2010) AMPA receptors gate spine $\mathrm{Ca}(2+)$ transients and spike-timing-dependent potentiation. Proc Natl Acad Sci U S A 107(36):15975-15980.

7. Gulledge AT, Carnevale NT, Stuart GJ (2012) Electrical advantages of dendritic spines. PLoS One 7(4):e36007.

8. Desmond NL, Levy WB (1986) Changes in the postsynaptic density with long-term potentiation in the dentate gyrus. J Comp Neurol 253(4):476-82.

9. Ehrlich I, Malinow R (2004) Postsynaptic density 95 controls AMPA receptor incorporation during long-term potentiation and experience-driven synaptic plasticity. J Neurosci 24(4):916-927.

10. Patterson MA, Szatmari EM, Yasuda R (2010) AMPA receptors are exocytosed in stimulated spines and adjacent dendrites in a Ras-ERK-dependent manner during long-term potentiation. Proc Natl Acad Sci U S A 107(36):15951-6.

11. Makino H, Malinow R (2009) AMPA receptor incorporation into synapses during LTP: the role of lateral movement and exocytosis. Neuron 64(3):381-90.

12. Ashby MC, Maier SR, Nishimune A, Henley JM (2006) Lateral diffusion drives constitutive exchange of AMPA receptors at dendritic spines and is regulated by spine morphology. $J$ Neurosci 26(26):7046-7055.

13. Tønnesen J, Katona G, Rózsa B, Nägerl UV (2014) Spine neck plasticity regulates compartmentalization of synapses. Nat Neurosci 17(5):678-85.

14. Wang L, Dumoulin A, Renner M, Triller A, Specht CG (2016) The Role of Synaptopodin in Membrane Protein Diffusion in the Dendritic Spine Neck. PLoS One 11(2):e0148310.

15. Matsuzaki M, Honkura N, Ellis-Davies GCR, Kasai H (2004) Structural basis of long-term potentiation in single dendritic spines. Nature 429(6993):761-766. 
16. Oertner TG (2009) How do synapses measure milliseconds? Front Comput Neurosci 3:7.

17. Svoboda K, Tank DW, Denk W (1996) Direct measurement of coupling between dendritic spines and shafts. Science 272(5262):716-719.

18. Bloodgood BL, Sabatini BL (2005) Neuronal activity regulates diffusion across the neck of dendritic spines. Science 310(5749):866-869.

19. Noguchi J, Matsuzaki M, Ellis-Davies GC, Kasai H (2005) Spine-neck geometry determines NMDA receptor-dependent Ca2+ signaling in dendrites. Neuron 46(4):609-622.

20. Bartol TM, et al. (2015) Nanoconnectomic upper bound on the variability of synaptic plasticity. Elife 4(NOVEMBER2015):e10778.

21. Harvey CD, Svoboda K (2007) Locally dynamic synaptic learning rules in pyramidal neuron dendrites. Nature 450(7173):1195-1200.

22. Tanaka J, et al. (2008) Protein synthesis and neurotrophin-dependent structural plasticity of single dendritic spines. Science 319(5870):1683-1687.

23. Nevian T, Sakmann B (2004) Single spine Ca2+ signals evoked by coincident EPSPs and backpropagating action potentials in spiny stellate cells of layer 4 in the juvenile rat somatosensory barrel cortex. J Neurosci 24(7):1689-1699.

24. Beaulieu-Laroche L, Harnett MT (2018) Dendritic Spines Prevent Synaptic Voltage Clamp. Neuron 97(1):75-82.e3.

25. Kusters R, Kapitein LC, Hoogenraad CC, Storm C (2013) Shape-Induced Asymmetric Diffusion in Dendritic Spines Allows Efficient Synaptic AMPA Receptor Trapping. Biophys J 105(12):2743-2750.

26. Palmer LM, Stuart GJ (2009) Membrane potential changes in dendritic spines during action potentials and synaptic input. J Neurosci 29(21):6897-903.

27. Ngo-Anh TJ, et al. (2005) SK channels and NMDA receptors form a Ca2+-mediated feedback loop in dendritic spines. Nat Neurosci 8(5):642-649.

28. Gee CE, Ohmert I, Wiegert JS, Oertner TG (2017) Preparation of Slice Cultures from Rodent Hippocampus. Cold Spring Harb Protoc 2017(2):pdb.prot094888.

29. Campbell RE, et al. (2002) A monomeric red fluorescent protein. Proc Natl Acad Sci U S A 99(12):7877-7882.

30. Patterson GH, Lippincott-Schwartz J (2002) A photoactivatable GFP for selective photolabeling of proteins and cells. Science 297(5588):1873-7.

31. Pologruto TA, Sabatini BL, Svoboda K (2003) Scanlmage: Flexible software for operating laser scanning microscopes. Biomed Eng Online 2(1):13.

32. Suter BA, et al. (2010) Ephus: multipurpose data acquisition software for neuroscience experiments. Front Neural Circuits 4(August):1-12. 


\section{Figures}
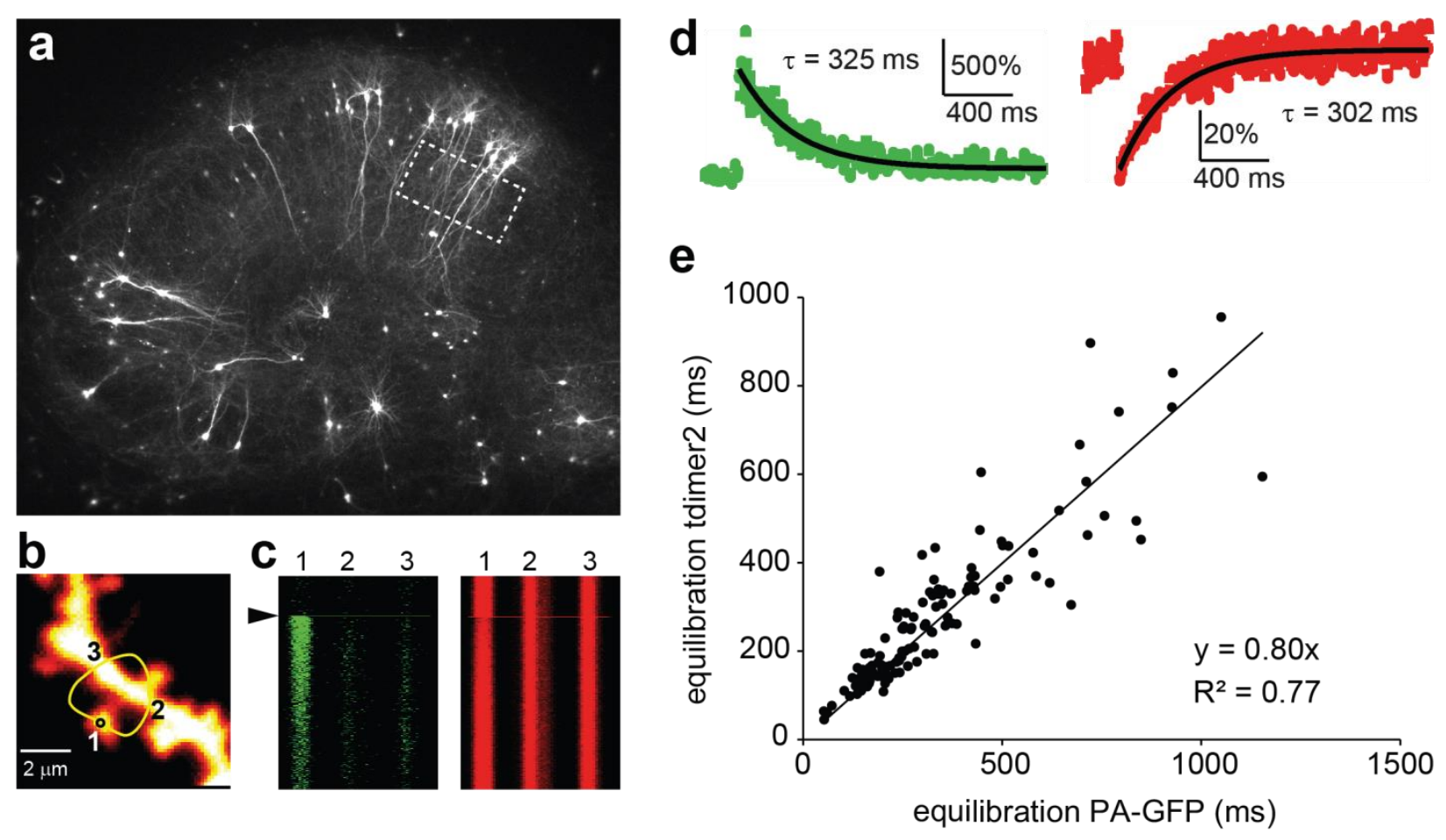

Figure 1. Measuring diffusional coupling between spine and parent dendrite. (a) Organotypic hippocampal culture after gene gun transfection with tdimer2 / PA-GFP. Box marks region of interest. (b) Spiny dendrite of CA1 pyramidal cell expressing tdimer2 / PAGFP at high magnification. Yellow line indicates user-defined scan path $(980 \mathrm{~nm})$ across spine head $(1)$ and dendrite $(2,3)$. Black circle indicates position of photoactivation pulse $(810 \mathrm{~nm})$. (c) At $t=200 \mathrm{~ms}$, a brief laser pulse $(810 \mathrm{~nm})$ was directed to the spine head (region 1) to simultaneously activate PA-GFP (left) and bleach tdimer2 (right). (d) The decay time course of PA-GFP fluorescence in the spine head (green) was very similar to the time course of fluorescence recovery after photobleaching (FRAP) of tdimer2 (red). Black lines show single exponential fits. (e) For individual spines, the decay time constant of PA-GFP and recovery time constant of tdimer 2 were highly correlated ( $n=120$ spines, 15 cells). The distribution of $\tau$ is very broad and skewed towards strongly coupled spines (median $\tau_{\text {PA-GFP }}=275 \mathrm{~ms}$, range: $52 \mathrm{~ms}-1154 \mathrm{~ms}$, median $\tau_{\text {tdimer2 }}$ $=250 \mathrm{~ms}$, range: $45 \mathrm{~ms}-955 \mathrm{~ms}$ ). 

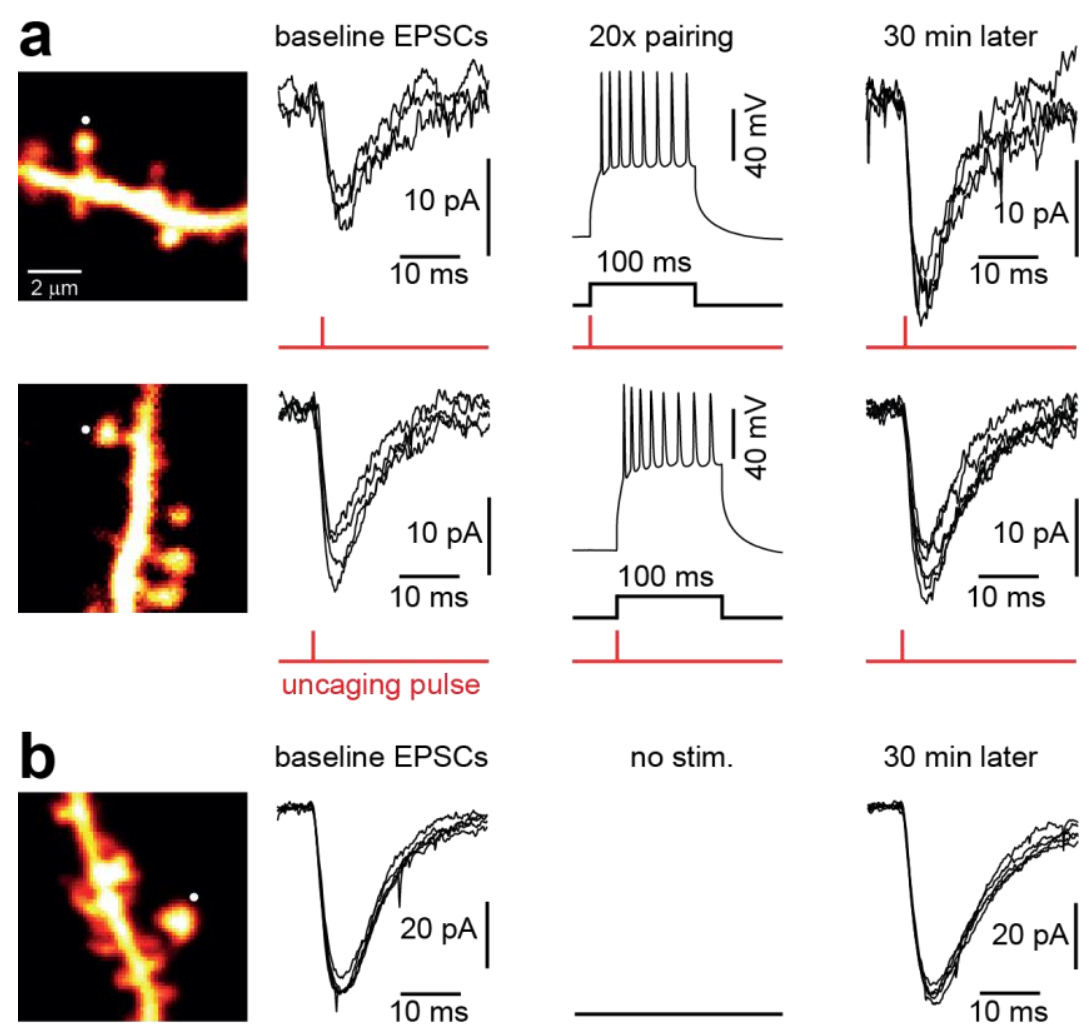

baseline EPSCs

no stim.

30 min later
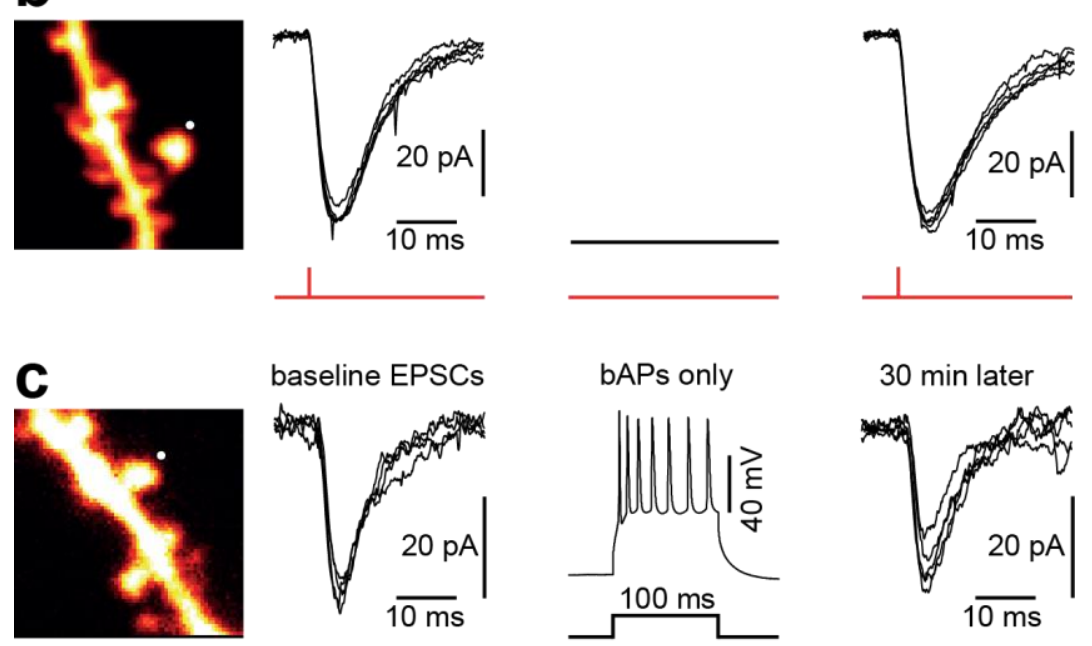

baseline EPSCs

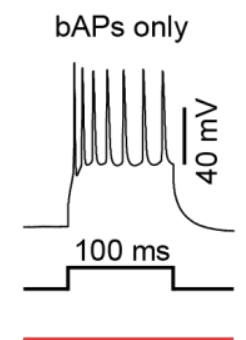

30 min later
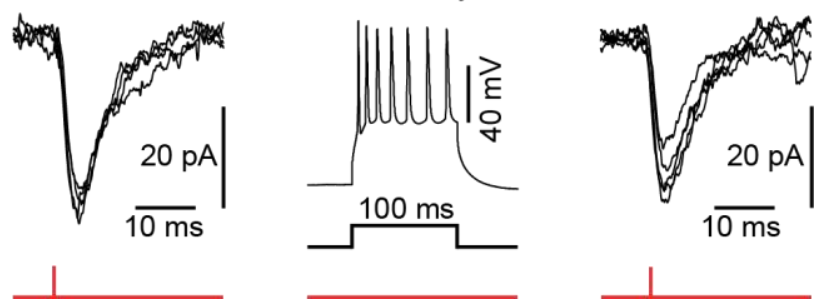

Figure 2. Plasticity induction by pairing of glutamate uncaging with postsynaptic depolarization. (a) Glutamate uncaging on a single spine (interval = $1 \mathrm{~min}$ ) caused uEPSCs of consistent amplitude. Uncaging pulses were paired with current injections into the postsynaptic neuron (20 pairings at $0.5 \mathrm{~Hz}$ ). Thirty minutes after the pairing protocol, UEPSCs were potentiated in some spines (upper row) whereas no plasticity occurred in other spines (lower row). (b) Control experiment without pairing. (c) Control experiment with only postsynaptic current injections, causing bursts of bAPs (20 repeats at $0.5 \mathrm{~Hz}$ ). 

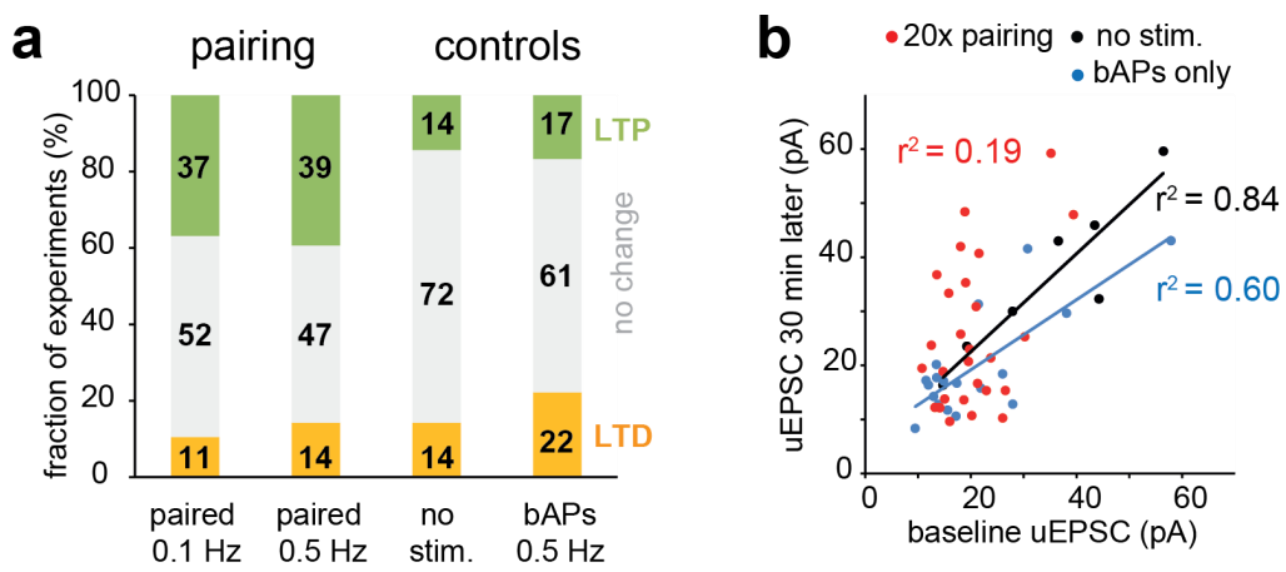

Figure 3. Analysis of plasticity experiments. (a) Outcome of uncaging-pairing stimulation and control experiments. Green indicates significant potentiation, gray indicates no change, and orange indicates significant depression. Numbers indicate \% of experiments with the specified outcome (paired $0.1 \mathrm{~Hz}: n=$ 19; paired $0.5 \mathrm{~Hz}: n=28$; no stimulation: $n=7$; bAPs: $n=18$ ). (b) Under control conditions, uEPSC amplitudes at baseline and 30 min later were highly correlated (black markers, $n=7$ ). Repeated action potential bursts triggered some changes, but baseline and late uEPSCs were still correlated (blue markers, $n=18$ ). The pairing protocol decorrelated baseline and final potency of the synapses (red markers, $n=28$ ). 

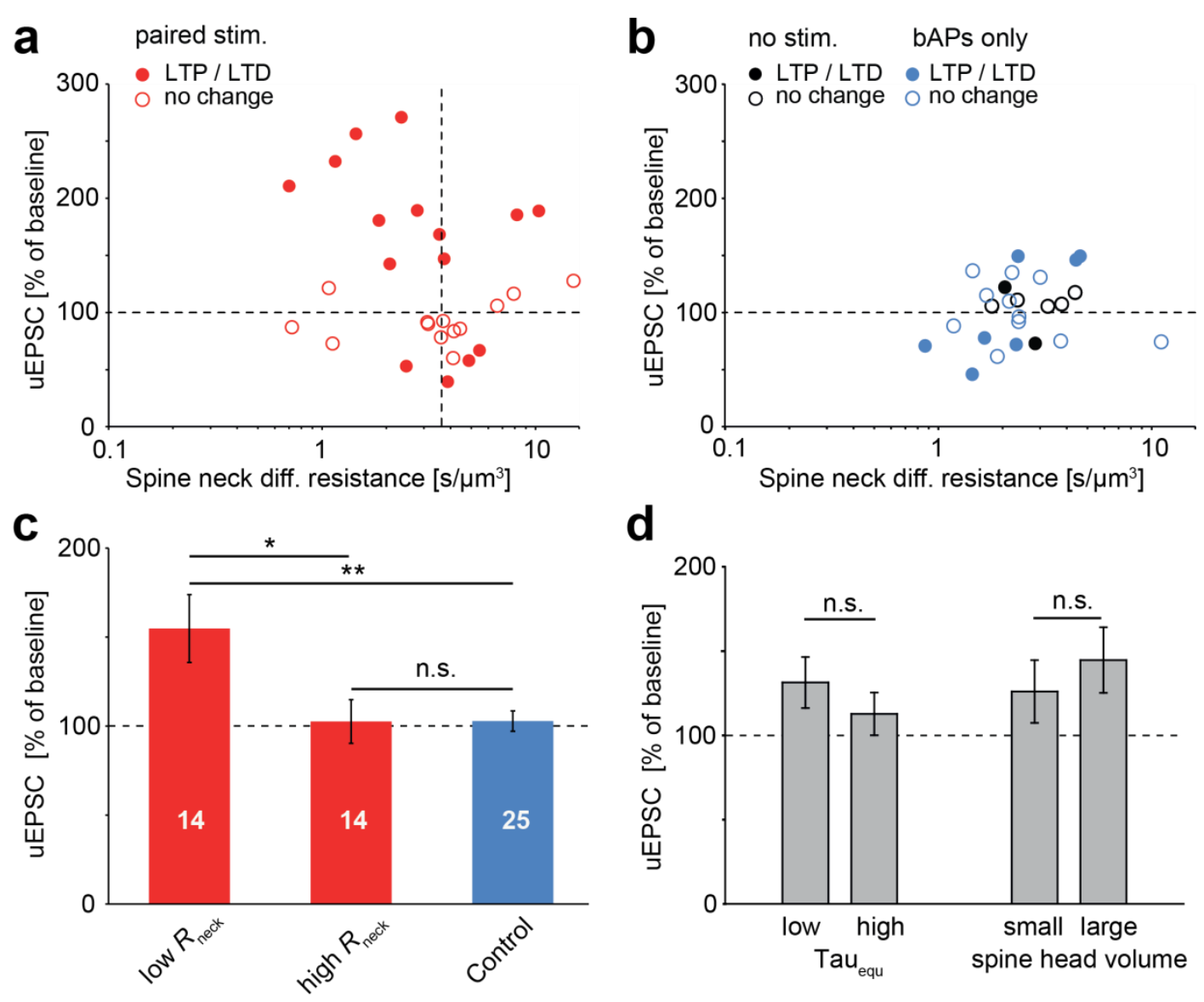

Figure 4. Impact of spine neck resistance on the induction of long-term plasticity. (a) Relationship between spine neck resistance and outcome of plasticity experiments. Filled symbols indicate experiments where uEPSC were significantly different before and after pairing at $0.5 \mathrm{~Hz}(\mathrm{p}<0.05)$, open symbols indicate experiments with no change of UEPSC amplitude. Dotted vertical line indicates median spine neck resistance of paired spines. (b) Unpaired control spines without stimulation (black) or twenty bursts of back-propagating action potentials at $0.5 \mathrm{~Hz}$ (blue). (c) Spines were split into two groups at the median $R_{\text {neck }}$ (see panel $A$, vertical dashed line). Synapses on low $R_{\text {neck }}$ spines were significantly potentiated compared to synapses on high $\mathrm{R}_{\text {neck }}$ spines $(p<0.05)$ and controls $(p<0.01)$. Synapses on high $R_{\text {neck }}$ spines were not significantly different from the unpaired controls (from panel $b$ ). (d) Splitting the population of paired spines by the median time constant of equilibration ( $\tau_{\text {equ }}$ ) or by the median volume of the spine head did not reveal significant differences between groups (low vs. high $\tau_{\text {equ }}, P=0.8$; small vs. large spine heads, $P=0.2$ ). 